\title{
Nuevas citas de flora amenazada y rara en el País Vasco III.
}

\section{New records of threatened and rare flora in the Basque Country III.}

Juan Manuel Pérez de Ana ${ }^{1 *}$

(6)

\section{Resumen}

Se ofrecen 48 citas interesantes desde el punto de vista corológico de 40 especies de plantas amenazadas y/o raras en el País Vasco (Bizkaia y Araba); 5 de ellas están protegidas en la Comunidad Autónoma del País Vasco.

Palabras clave: Álava-Araba, Bizkaia, conservación de la biodiversidad, especies amenazadas.

\begin{abstract}
48 observations of chorological interest of 40 threatened and/or rare plant species in the Basque Country (Bizcay and Araba) are offered; 5 of these plants are protected in the Autonomous Region of the Basque Country.
\end{abstract}

Key words: Álava-Araba, Bizkaia, biodiversity conservation, species of concern.

\section{Laburpena}

Honakoan, korologiaren ikuspuntutik interesgarri diren Euskal Herriko (Bizkaia eta Araba) 40 landare espezie mehatxatu edo/eta arraroren 48 aipamen aurkezten dira; horietatik 5 babestuta daude Euskal Autonomia Erkidegoan.

Gako hitzak: Álava-Araba, Bizkaia, biodibertsitatearen kontserbazioa, espezie mehatxatuak.

1 Diputación Foral de Bizkaia.

Departamento de Sostenibilidad y Medio Natural.

Lehendakari Aguirre, 9 - 2 48014 Bilbao.

*Correspondencia: perezdeana@gmail.com 
Se añaden 48 citas nuevas de 40 especies de flora amenazada y rara en el País Vasco (Bl: Bizkaia y Vl: Araba), 5 de ellas incluidas en el Catálogo Vasco de Especies Amenazadas de la Comunidad Autónoma del País Vasco (Orden de 10 de enero de 2011) (https://www.euskadi.eus/y22-bopv/es/bopv2/datos/2011/02/1100937a.pdf), obtenidas en el período 2009-2021. En su caso, se ofrecen los datos obtenidos del censo de sus poblaciones con el fin de que sirvan a su conservación. Los táxones tratados son aquellos que en Aseginolaza et al. (1985) no se citaron o se dieron como raros o muy raros para la provincia, pero también hay otros más comunes, pero que se citan por primera vez para la parte vasca de Sierra Sálvada, en otro caso es una segunda cita y en un último caso se da cuenta de una gran población de un taxon escaso. Para cada cita se señala: municipio, paraje, cuadrícula UTM 1x1 km en Datum ETRS89, altitud, hábitat y fecha. Todas las citas están incluidas en el huso 30T y, más en concreto, en 12 cuadrículas UTM de 10x10 km: VN76, VN86, VN89, VN95, VN96, WN05, WN06, WN07, WN15, WN18, WN26 y WN27. Se ha proporcionado al Servicio de Patrimonio Natural del Departamento de Medio Ambiente de la Diputación Foral de Bizkaia información geográfica de alta precisión de estos hallazgos. Se ha seguido la taxonomía de Flora iberica (www.floraiberica.es), accesible a través de la página del Real Jardín Botánico (https://bibdigital.rjb.csic.es/records/item/9895-flora-iberica?offset=1).

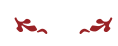

\section{Adonis vernalis $\mathrm{L}$.}

(BI) Orduña, Lendoño Goikoa, VN9462, 620-670 m, unas 75 plantas en plena floración en una ladera soleada, 15.04.2021.

Descubierta por Dorleta Albizua. Se aporta una cuarta localidad para la vertiente cantábrica de Sierra Sálvada (Pérez de Ana, 2008, 2010).

\section{Allium ursinum L. subsp. ursinum}

(VI) Aiara, Aguiñiga, VN9263, 800-820 m, una densa población de 120x80 m, en el borde superior del hayedo, 15.04.2021.

Primera cita para la parte alavesa de Sierra Sálvada. En el año 2009 se descubre la primera población de la parte vasca de Sierra Sálvada, en Orduña (Pérez de Ana, 2010).

\section{Anemone ranunculoides $\mathrm{L}$.}

(VI) Aiara, Kobata, VN8963, 1060 m, varios centenares de plantas en el borde de un hayedo en dos poblaciones separadas por unos 90 metros, 29.04.2021 (Fig. 1A).

Desconocida de Sierra Sálvada y su entorno. Dentro del País Vasco Aseginolaza et al. (1985) la herborizaron únicamente en (VI) Foronda, WN2249, 550 m, de donde posteriormente desapareció tras que talaran la chopera donde vivía. Alejandre et al. 


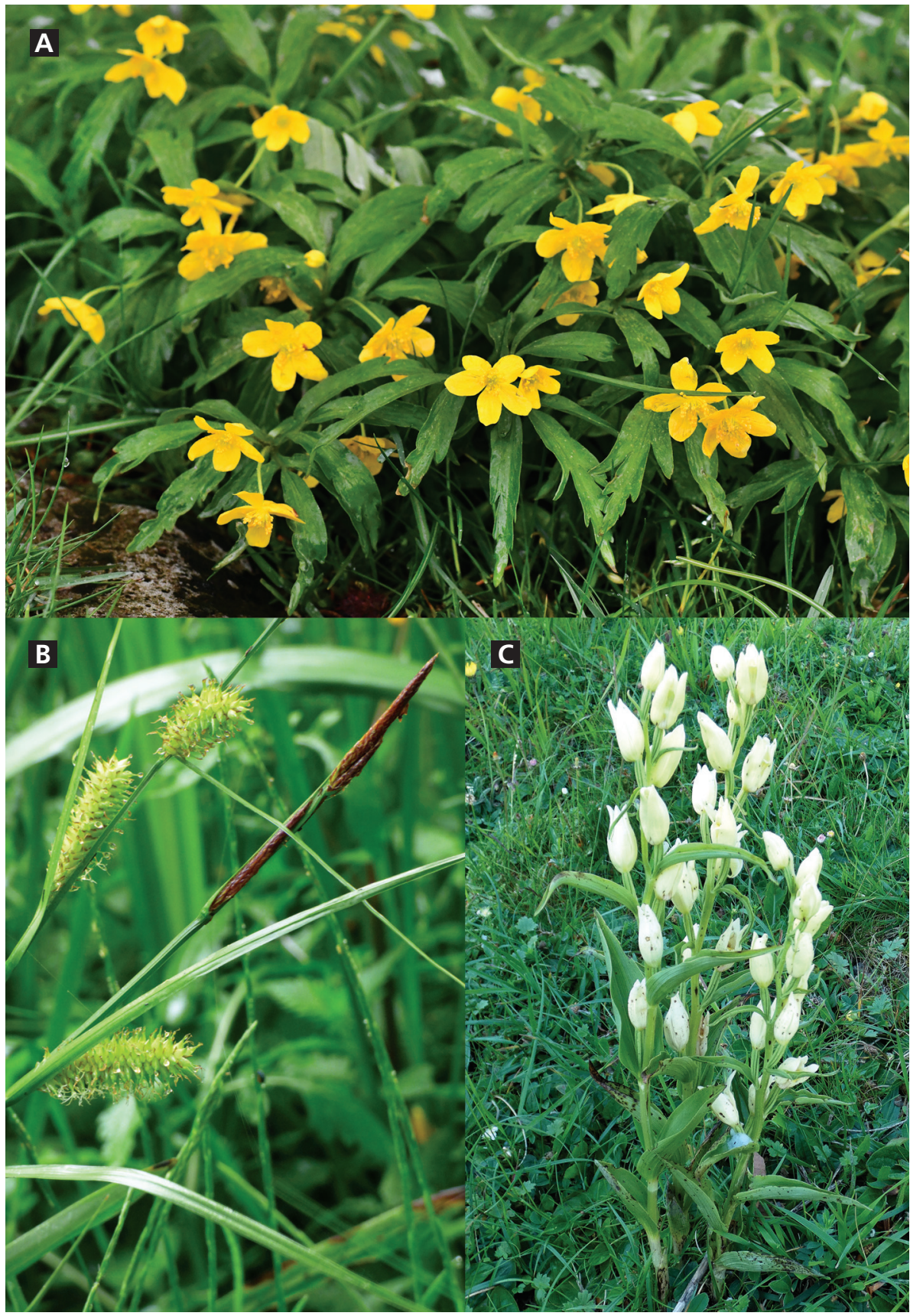

Fig.- 1. (A) Anemone ranunculoides L., (B) Carex vesicaria L., (C) Cephalanthera damasonium (Mill.) Druce. 
(1987) la citaron de la orilla del río Bayas cerca de Sendadiano, WN0749, 550 m, y de la sierra de Entzia, en Peña Caída, WN6244, 1080 m.

\section{Arabis turrita L.}

(BI) Lemoa, Arlanpe, WN1981, 320 m, a la sombra de unos árboles en una cresta caliza. 25.05.2020.

En Bizkaia solo se conocía de los montes del Duranguesado: Mañaria, WN2676, 440 m, y Eskubaratz, WN2673, 740 m (Aseginolaza et al., 1985).

Arenaria montana L. subsp. montana

(VI) Aiara, Vallevicencio, VN9061, 950 m, 11.05.2021.

Desconocida de Sierra Sálvada y su entorno (Aseginolaza et al., 1985). Sin duda, las abundantes precipitaciones lavan las bases de los suelos calcáreos de esta sierra y permiten la presencia de esta especie acidófila.

\section{Astragalus glycyphyllos L.}

(BI) Orduña, Atxondo, VN9862, 470 m, 11.06.2015.

Aseginolaza et al. (1985) la herborizaron muy cerca, en Orduña, VN9760, 450 m. Hace unos 20 años existía una población que desapareció y desde entonces no se había vuelto a encontrar en Sierra Sálvada y su entorno.

\section{Carex vesicaria L.}

(BI) Zeanuri, Saldropo, WN2266, 610 m, en remanso de arroyo donde abundaba Limniris pseudacorus, 28.05.2018 (Fig. 1B).

Segunda población en el Macizo de Gorbeia de una planta muy rara en el País Vasco que vive en orillas de charcas y arroyos remansados sobre sustrato silíceo. Aseginolaza et al. (1985) la citaron de (BI) Otxandio, WN2864, $550 \mathrm{~m}$.

\section{Carpinus betulus L.}

(BI) Bedia, Jauregi, WN1684, 80-120 m, 6 individuos con unos 5 vástagos cada uno que nacen de cepa, 27.04.2021.

Descubierta por Aritz Oregui. Se descubrió un único árbol en Bizkaia, en Lemoa, Lemoatx, WN1785, 210 m (Asensio et al., 1990), pero luego desapareció (Pérez de Ana, 2014). Ahora aparece una pequeña población a menos de $2 \mathrm{~km}$ de dicha localidad. Fernando Ruiz Moneo me ha informado de la existencia de varios ejemplares maduros en el jardín del Palacio de Bidekolea, localizados a unos 500 metros de distancia en línea de recta de los 6 individuos encontrados. Incluido en la categoría "en peligro de extinción" del Catálogo Vasco de Especies Amenazadas. 


\section{Cephalanthera damasonium (Mill.) Druce}

(BI) Orduña, Ponata, VN9362, 920 m, una única planta con tres tallos con flores, 27.05.2020 (Fig. 1C).

Descubierta por José Ignacio García Plazaola. Se trata de la primera observación de esta especie en Sierra Sálvada y la segunda en Bizkaia y en la vertiente cantábrica del País Vasco (Patino et al., 2010).

Cladium mariscus (L.) Pohl

(VI) Urkabustaiz, laguna de El Troncón, WN0857, 585 m, orilla de laguna, 17.08.2018. Desconocida del Macizo de Gorbeia, Sierra Sálvada y sus entornos (Aseginolaza et al., 1985).

\section{Geum sylvaticum Pourr.}

(BI) Orduña, monte Babio, VN9665, 380-410 m, varios cientos de ejemplares en quejigales orientados al sur, 09.04.2017.

(VI) Aiara, Lodares y Tremoledo, VN8961, VN8962 y VN9061, 940-1090, varios miles de ejemplares en ladera orientada al sur, 06.05.2021, 07.05.2021 y 11.05.2021.

Primera población de la parte alavesa de Sierra Sálvada y segunda población de Bizkaia tras la primera en Orduña, WN0260, 640 m (Aseginolaza et al., 1985).

Hesperis matronalis L. subsp. candida (Kit. ex Schulzer, Kanitz \& J. A. Knapp) Hegi \& Em. Schmid.

(VI) Zuia, Lukiano, WN1154, 600 m, común en la ribera del río Bayas, 20.06.2018.

Desconocida del Macizo de Gorbeia y su entorno (Aseginolaza et al., 1985).

Himantoglossum hircinum (L.) Spreng.

(BI) Artzentales, Alén, VN8290, 535 m, un solo ejemplar entre rocas calizas, 14.05.2017.

(BI) Orduña, Gama, VN9859, 290, dos ejemplares cerca de una balsa, 10.05.2021.

(VI) Aiara, Maroño, VN9566, 330 m, 6 plantas en el arcén de una carretera, 26.05.2020 y 3 plantas el 07.05.2021.

Descubierta por Angélica Llaguno en Alén. Se encontró recientemente una nueva población de esta especie en el arcén de una carretera (Pérez de Ana, 2017), donde los desbroces periódicos favorecen a varias especies de orquídeas. Incluido en la categoría "rara" del Catálogo Vasco de Especies Amenazadas.

\section{Marrumbium vulgare L.}

(BI) Orduña, Lendoñobeiti, VN9762, 350 m, 08.04.2017. 
Ya se conocía de (BI) Orduña, VN9960, 270 m (Aseginolaza et al., 1985), pero, aunque se ha buscado activamente en los ambientes adecuados, se ha tardado varios años en redescubrirla.

\section{Myriophyllum verticillatum $\mathrm{L}$.}

(VI) Aiara, balsa de La Lastrilla, VN9162, 1.130 m, 01.09.2017.

No se conocía de Sierra Sálvada (Aseginolaza et al., 1985).

\section{Neotinea maculata (Desf.) Stearn}

(VI) Aiara, Lodares, VN9062, 1070 m, 1 planta en ladera orientada al sur, 11.05.2021.

(VI) Aiara, Tremoledo, VN9061, 990 m, 6 plantas en ladera orientada al sur, 11.05.2021.

No se conocía de la parte vasca de Sierra Sálvada (Aseginolaza et al., 1985).

\section{Ophioglossum vulgatum $\mathrm{L}$.}

(BI) Dima, Kaltzadea, WN2075, 330 m, una población 10x2 m en el borde de un camino antiguo, 10.04.2021.

Descubierta por Joserra Undagoitia en abril de 2020, en una localidad intermedia entre las conocidas en el Parque Natural de Gorbeia (Pérez de Ana, 2017) y en el de Urkiola (Díaz et al., 2018). Incluido en la categoría "rara" del Catálogo Vasco de Especies Amenazadas.

\section{Orchis italica Poir.}

(VI) Amurrio, Larrinbe, WN0165, 320 m, un ejemplar en el arcén de una carretera, 12.05.2018.

Descubierta por Igor Martínez. No se conocía de la vertiente cantábrica del País Vasco ni del resto de la vertiente cantábrica de la Península lbérica (Aseginolaza et al., 1985). Incluido en la categoría "vulnerable" del Catálogo Vasco de Especies Amenazadas.

\section{Orchis morio L.}

(VI) Aiara, Lodares y Tremoledo, VN8961, VN8962 y VN9061, 930-1060 m, varios miles de plantas en ladera orientada al sur, 06.05.2021, 07.05.2021 y 10.05.2021.

Desconocida de la parte vasca de Sierra Sálvada (Aseginolaza et al. 1985). Todos los ejemplares observados se corresponden con lo que algunos autores llaman Orchis champagneuxii, que sería una especie con pocas flores, con el labelo netamente plegado, con la zona central blanca y sin manchas y los lóbulos laterales de un rosa claro. 
Orchis provincialis Balb. ex Lam \& DC.

(BI) Orduña, Garetxeta, VN9759, 500 m, 171 plantas con flores, 27.04.2017.

(VI) Aiara, Lodares, VN8961, 960 m, 7 plantas en ladera orientada al sur, 07.05.2021 En Orduña es una especie frecuente (Pérez de Ana, 2008), pero esta población destaca por su abundancia. No se conocía de la parte alavesa de Sierra Sálvada (Aseginolaza et al. 1985).

\section{Ornithogalum pyrenaicum L.}

(VI) Zuia, Lukiano, WN1154, 600 m, frecuente en la ribera del río Bayas, 20.06.2018. Desconocida del Macizo de Gorbeia y su entorno (Aseginolaza et al., 1985).

\section{Papaver dubium L.}

(VI) Amurrio, portillo de Pico del Fraile, VN9657, 800 m, pie de cantil, 03.06.2018. Segunda localidad de Sierra Sálvada y su entorno tras la de Aseginolaza et al. (1985) en (BI) Orduña, WN0360, 610 m.

\section{Pinguicula lusitanica L.}

(BI) Orozko, Iturburueta, WN0570, 580 m, zonas turbosas en manantial, 08.03.2021.

(BI) Orozko, Larragorri, WN0371, 670 m, zonas turbosas en manantial, 28.04.2021. Descubierta recientemente en (BI) Orozko, Jesuri, WN0472, 610-640 m (Díaz et al., 2018). Se añaden por tanto dos nuevas poblaciones en este macizo silíceo. Incluido en la categoría "rara" del Catálogo Vasco de Especies Amenazadas.

Polygonatum multiflorum (L.) All.

(BI) Dima, Presazelai, WN2675, 550 m, 263 plantas en la ribera del río Urkiola, 25.05.2018.

Gran población de una especie muy escasa en la zona (Pérez de Ana, 2017). En estos megaforbios también contamos 172 plantas florecidas de Valeriana pyrenaica, además de Euphorbia hyberna subsp. hyberna y otras especies.

\section{Potamogeton natans $\mathrm{L}$.}

(BI) Orduña, Bigandi, WN0360, 670 m, en un tramo de pista forestal actualmente inundado permanentemente de forma natural y cercado para la protección de una población de rana ágil (Rana dalmatina) que aquí se reproduce, 09.08.2018.

Desconocida de Sierra Sálvada y su entorno (Aseginolaza et al., 1985).

\section{Ranunculus hederaceus $\mathrm{L}$.}

(BI) Zeanuri, Saldropo, WN2266, 615 m, una población de 1 metro cuadrado en un arroyo, 17.05.2018. 
Muy rara en el País Vasco. Willkomm \& Lange (1880) la citaron de Bilbao y Portugalete. Aseginolaza et al. (1985) solo la herborizaron en una acequia en (VI) Aiara, Zuaza, vN9774, $200 \mathrm{~m}$.

\section{Ranunculus paludosus Poir.}

(VI) Aiara, Lodares, VN9061, 960-980 m, varios centenares de plantas en ladera orientada al sur, 07.05.2021.

Desconocida de la parte vasca de Sierra Sálvada (Aseginolaza et al. 1985).

Rhamnus pumila Turra subsp. pumila

(BI) Orduña, entre Atezabal y Bedarbide, VN9560, 980 m, 13.05.2009.

(BI) Orduña, portillo de la Barrerilla, VN9362, 900 m, 24.06.2014.

Primeras citas de Sierra Sálvada. Solo se conocía una cita de Bizkaia, en Abadiño, Mendiola, WN2974, 450 m (Aseginolaza et al., 1985).

\section{Rhynchospora alba (L.) Vahl}

(BI) Orozko, Algorta, WN1367, 820 m, en turberas, 04.09.2018.

Segunda población descubierta en el Macizo de Gorbeia tras la que encontraron Patxi Heras y Marta Infante en (BI) Areatza, Larreder, WN1670, 765 m (Orbela Elkartea, 2001).

\section{Sanguisorba officinalis $\mathrm{L}$.}

(BI) Aiara, pico del Aro, VN7964, 860 m, una única planta, 05.09.2018.

Desconocida de Sierra Sálvada y su entorno (Aseginolaza et al., 1985).

\section{Silene latifolia Poir.}

(BI) Orduña, Santa Clara, VN9959, 280 m, varios ejemplares en un herbazal al borde de un camino, 18.05.2018.

Desconocida de la parte vasca de Sierra Sálvada. Aseginolaza et al. (1985) la consideraron escasa y exclusiva de pies de roquedo y pedregales calizos de la vertiente mediterránea del País Vasco. Posteriormente, Patino \& Valencia (2000) la citaron como ruderal en la vertiente cantábrica en márgenes de autovía y carretera en Portugalete, Erandio y Bilbao (Bizkaia).

Silybum marianum (L.) Gaertn.

(VI) Aiara, Zubiaga, VN9465, 325 m, en un pastizal mal cuidado, 24.05.2020.

(BI) Orduña, San Antón, VN9760, 465 m, en una antigua escombrera, 24.05.2020. 
Guinea (1953) lo registró en Orduña, pero no se han publicado citas posteriores en Sierra Sálvada y su entorno.

\section{Sisyrinchium rosulatum E. P. Bicknell}

(BI) Orozko, Lezarrede, WN1472, 600 m, varios ejemplares en un pastizal dentro del Parque Natural de Gorbeia, 06.05.2017.

Originaria del sureste de Norteamérica. Desconocida del País Vasco hasta las citas de Cadiñanos et al. (2015) en Muskiz, monte Mello, VN8994, 95 m y de Novoa-Fariñas (2018) en Elorrio, Besaide, WN3771, $560 \mathrm{~m}$.

\section{Sorbus torminalis (L.) Crantz}

(BI) Orduña, Lendoñobeiti, VN9862, 425 m, 2 ejemplares, 22.11.2016.

Desconocida en Sierra Sálvada (Aseginolaza et al., 1985).

Sparganium erectum L. subsp. neglectum (Beeby) Schinz \& Thell.

(BI) Orduña, Bigandi, WN0360, 670 m, en un tramo de pista forestal actualmente inundado permanentemente de forma natural y cercado para la protección de una población de rana ágil que aquí se reproduce, 09.08.2018.

Desconocida de Sierra Sálvada y su entorno (Aseginolaza et al., 1985).

Stegnogramma pozoi (Lag.) K. Iwats.

(BI) Orozko, Lezeaga erreka, WN0676, 260 m, varios ejemplares en borde de arroyo, 04.01.2018.

Poblaciones en un nuevo arroyo afluente del río Nervión (véanse Barredo, 1996 y Pérez de Ana, 2014 y 2017).

Tulipa sylvestris L. subsp. australis (Link) Pamp.

(VI) Aiara, Lodares, VN8962, 1020 m, 3 plantas en ladera orientada al sur, 07.05.2021. Desconocida de la parte vasca de Sierra Sálvada (Aseginolaza et al. 1985).

\section{Typha domingensis Pers.}

(BI) Orduña, La Rondina, VN9859, 290 m, en la orilla de una balsa, 13.08.2018.

Desconocida de Sierra Sálvada y su entorno (Aseginolaza et al., 1985).

\section{Urtica urens $\mathrm{L}$.}

(VI) Amurrio, portillo de Pico Fraile, VN9657, 790 m, pie de cantil estercolado por cabras, 03.06.2018.

Desconocida de Sierra Sálvada y su entorno (Aseginolaza et al., 1985). 


\section{Valeriana tuberosa L.}

(VI) Aiara, Lodares, VN9062. 1070 m, una sola población compuesta por unas 100 plantas, 11.05.2021.

Desconocida de Sierra Sálvada y su entorno (Aseginolaza et al., 1985).

\section{Bibliografía}

Alejandre, J. A., Morante, G., Uribe-Echebarria, P. M., Urrutia, P., 1987. Notas corológicas sobre la flora vascular del País Vasco y aledaños (I). Est. Inst. Alavés de la Naturaleza 2, 205-212.

Aseginolaza Iparragirre, C., Gómez García, D., Lizaur Sukia, X., Montserrat Martí, G., Morante Serrano, G., Salaverría Monfort, M. R., Uribe-Echebarría Díaz, P. M., Alejandre Sáenz, J. A., 1985. Catálogo Florístico de Álava, Vizcaya y Guipúzcoa. Servicio Central de Publicaciones del Gobierno Vasco, Vitoria-Gasteiz.

Asensio González, R., Francés Arriola, E., Ortega Movillo, C., Vadillo Robredo, J.M. 1990. Árboles singulares de Euskadi. Servicio Central de Publicaciones del Gobierno Vasco. Vitoria-Gasteiz.

Barredo Pérez, J. J., 1996. Nuevos datos sobre la distribución y el hábitat de algunas plantas termófilas oceánicas en el tramo oriental de la Cornisa Cantábrica. Munibe, Cienc. nat 48, 39-48.

Cadiñanos, J.A., Llorente-Rodrigo, A., Fidalgo-Prieto, E., 2015. Nuevas aportaciones a la flora vascular en el País Vasco, Cantabria, Asturias y Burgos (norte de la Península Ibérica). Munibe, Cienc. nat. 63, 73-97.

Díaz, E. J., Elorza, J., González-Ahedo, S., Miguel, E., Orrantia, O., Patino, S., Prieto, A., Valencia, J., Zorrakin, B., 2018. Notas corológicas sobre la flora vascular del País Vasco y aledaños (XIII). Munibe, Cienc. nat. 66, 155-183.

Guinea, E., 1953. Geografía botánica de Santander. Diputación Provincial de Santander.

Novoa-Fariñas, I., 2018. Aportaciones al conocimiento de la distribución de algunas plantas poco frecuentes en el País Vasco. Munibe, Cienc. nat. 66, 257-274.

Orbela Elkartea, 2001. Caracterización de los Trampales de Larreder. Parque Natural de Gorbeia. Bizkaia. Informe inédito.

Patino, S., Valencia, J., 2000. Notas corológicas sobre la flora vascular del País Vasco y aledaños (IX). Est. Mus. Cienc. Nat. de Álava 15, 221-238.

Patino, S., Valencia, J., Miguel, E., Prieto, A., Elorza, J., Oyanarte, T., Toral, E., Otxoa, E., Díaz, E., 2010. Notas corológicas sobre la flora vascular del País Vasco y aledaños (XII). Est. Mus. Cienc. Nat. de Álava 23, 91-111.

Pérez de Ana, J. M., 2008. Nuevas citas de flora amenazada y rara en Sierra Salvada. Est. Mus. Cienc. Nat. de Álava 22, 85-100. 
Pérez de Ana, J. M., 2010. Nuevas citas de flora amenazada y rara en Sierra Salvada, II. Est. Mus. Cienc. Nat. de Álava 23, 61-66.

Pérez de Ana, J. M., 2014. Nuevas citas de flora amenazada y rara en el País Vasco. Munibe, Cienc. nat. 62, 103-117.

Pérez de Ana, J. M., 2017. Nuevas citas de flora amenazada y rara en el País Vasco II. Munibe Cienc. nat. 65, 127-140.

Willkomm, H. M., Lange, J., 1880. Prodromus Florae Hispanicae. Vol. 3E. Schweizerbart, Stuttgart.

Fecha de recepción/ Date of reception: 17/05/2021

Fecha de aceptación / Date of acceptance: 23/10/2021

Editor Asociado / Associate editor: Cristina Herrero-Jáuregui 